\title{
Cavitary Lung Lesion in a Patient with Systemic Lupus Erythematosus: An Unusual Manifestation of Cytomegalovirus Pneumonia
}

\author{
Sihyung Park , Hang Jea Jang ${ }^{1}$, Yang Wook Kim, Bong Soo Park , Hyun Kuk Kim ${ }^{1}$, Jong Woon Song ${ }^{2}$, \\ Yeon Mi Kim ${ }^{3}$, Kyubok Jin ${ }^{1}$ \\ Departments of ${ }^{1}$ Internal Medicine, ${ }^{2}$ Radiology, and ${ }^{3}$ Pathology, Inje University Haeundae Paik Hospital, Inje University College of Medicine, \\ Busan, Korea
}

\begin{abstract}
Cytomegalovirus (CMV), a member of the human herpesvirus group, causes severe disease in immunocompromised patients. In particular, CMV pneumonia can be a life-threatening disease to patients taking immunosuppressive drugs. The radiographic manifestations of CMV are variable and may consist of reticular or reticulonodular patterns, ground-glass opacities, air-space consolidations, or mixed patterns. A cavitary lesion in pneumonia associated with CMV infection is extremely rare. Herein we report on a case of CMV pneumonia which presented with a cavitary lesion and was treated successfully in a systemic lupus erythematosus patient who was taking immunosuppressive drugs. (J Rheum Dis 2015;22:387-390)
\end{abstract}

Key Words. Pneumonia, Cytomegalovirus, Systemic lupus erythematosus

\section{INTRODUCTION}

In connective tissue disease such as systemic lupus erythematosus (SLE) [1], infections are important causes of morbidity and mortality. These infections are related to the result of immune abnormalities, organ system manifestations associated with primary diseases, and its treatment [1]. Cytomegalovirus (CMV) infection is a kind of opportunistic infection in immunocompromised patients. CMV infection is defined as detection of virus by CMV antigenemia test or quantitative polymerase chain reaction (PCR) and intranuclear inclusions by histologic examination [2]. Among the CMV infection, CMV pneumonia is a life-threatening opportunistic infection which have the radiologic manifestations of various patterns. It is hard to distinguish such infections from other diseases by pulmonary symptoms. Therefore, the mortality of CMV pneumonia is still high due to delayed treatment. Herein, we report a case of SLE patient with cavitary CMV pneu- monia and its successful treatment.

\section{CASE REPORT}

A 34-year-old woman visited our out-patients clinic with complaints of febrile sensation, cough and blood tinged sputum for four days. Three months ago, she had been diagnosed as having SLE complicated by lupus nephritis (World Health Organization class IV). Before her visit, she had been in stable conditions with taking low dose oral prednisolone and $1 \mathrm{~g}$ of mycophenolate mofetil for every day.

On her admission, her blood pressure was 90/60 $\mathrm{mmHg}$, body temperature was $38.2^{\circ} \mathrm{C}$, heart rate was 90 beats per minute; and respiratory rate was 22 breaths per minute. Physical examination revealed coarse breath sound on right upper lung field, and pale conjunctivae. Laboratory data showed a white blood cell count of $1,000 / \mathrm{mm}^{3}$ (neutrophil $80 \%$ ), hemoglobin of $7.2 \mathrm{~g} / \mathrm{dL}$,

Received : January 22, 2015, Revised : (1st) February 26, 2015, (2nd) March 20, 2015, (3rd) March 23, 2015, Accepted : March 23, 2015

Corresponding to : Kyubok Jin, Department of Internal Medicine, Inje University Haeundae Paik Hospital, Inje University College of Medicine, 875 Haeun-daero, Haeundae-gu, Busan 48108, Korea. E-mail : mdjin922@gmail.com 
hematocrit of $21.9 \%$, platelet count of $103,000 / \mathrm{mm}^{3}$, blood urea nitrogen of $28.0 \mathrm{mg} / \mathrm{dL}$, serum creatinine of $1.66 \mathrm{mg} / \mathrm{dL}$, and serum albumin of $2.6 \mathrm{~g} / \mathrm{dL}$. The level of C-reactive protein (CRP) was $0.3 \mathrm{mg} / \mathrm{dL}$. The titers of lupus activity factors had improved ( 37.9 to $103.5 \mathrm{mg} / \mathrm{dL}$ for C3, 8.9 to $24.4 \mathrm{mg} / \mathrm{dL}$ for $\mathrm{C} 4$ and 126.30 to 0.60 $\mathrm{IU} / \mathrm{mL}$ for anti-dsDNA antibody by immunoradiometric assay [reference range $<5.30 \mathrm{IU} / \mathrm{mL}$ ]), and proteinuria also improved from 11.6 to 1.2 using by spot urine protein to creatinine ratio. A chest X-ray showed a new cavitary lesion in right upper lung field compared to her prior chest X-ray done 2 weeks earlier (Figure 1). Computed tomography (CT) of the chest revealed a cavitary lesion

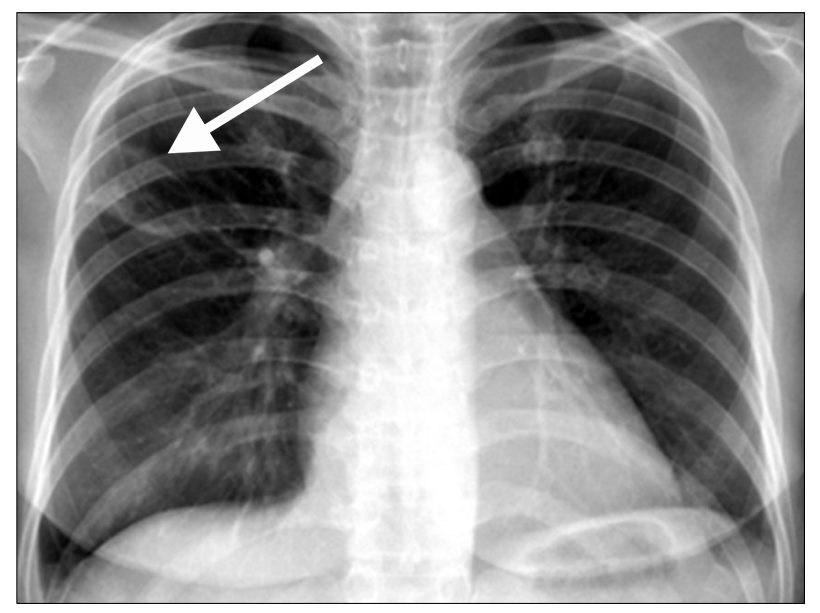

Figure 1. Chest plain radiography shows a large cavitary mass like lesion in the right upper lung field (arrow). with $40 \times 30 \times 40 \mathrm{~mm}$ in sized and $7 \mathrm{~mm}$ in thickness (Figure 2). Blood, urine, and sputum cultures for bacteria were negative. Tests for Mycobacterium tuberculosis were negative including QuantiFERON-TB (Cellestis Limited, Chadstone, Vic, Australia) test. Fungal tests and anti-neutrophil cytoplasm antibody were also negative. Viral tests were negative including CMV immunoglobulin (Ig)M. However, CMV antigenemia test (pp65Ag) and serum CMV PCR were positive (above 100 cells/200,000 leukocytes and 657,641 copies/mL, respectively). CT-guided transthoracic needle biopsy (TNB) was performed to obtain tissue confirmation. Histopathologic findings revealed multiple intranuclear inclusions in the pneumo-

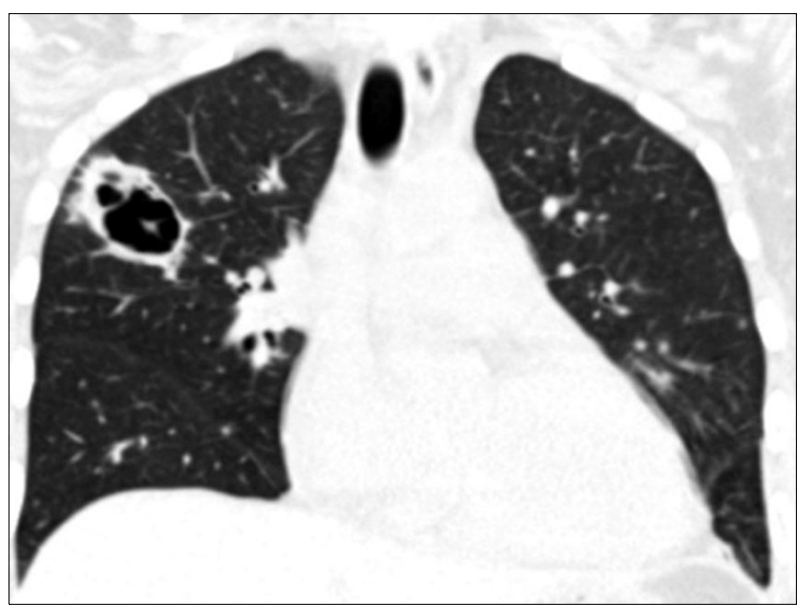

Figure 2. Chest computed tomography with coronal reformatted image shows a large cavitary mass like lesion in the right upper lobe.
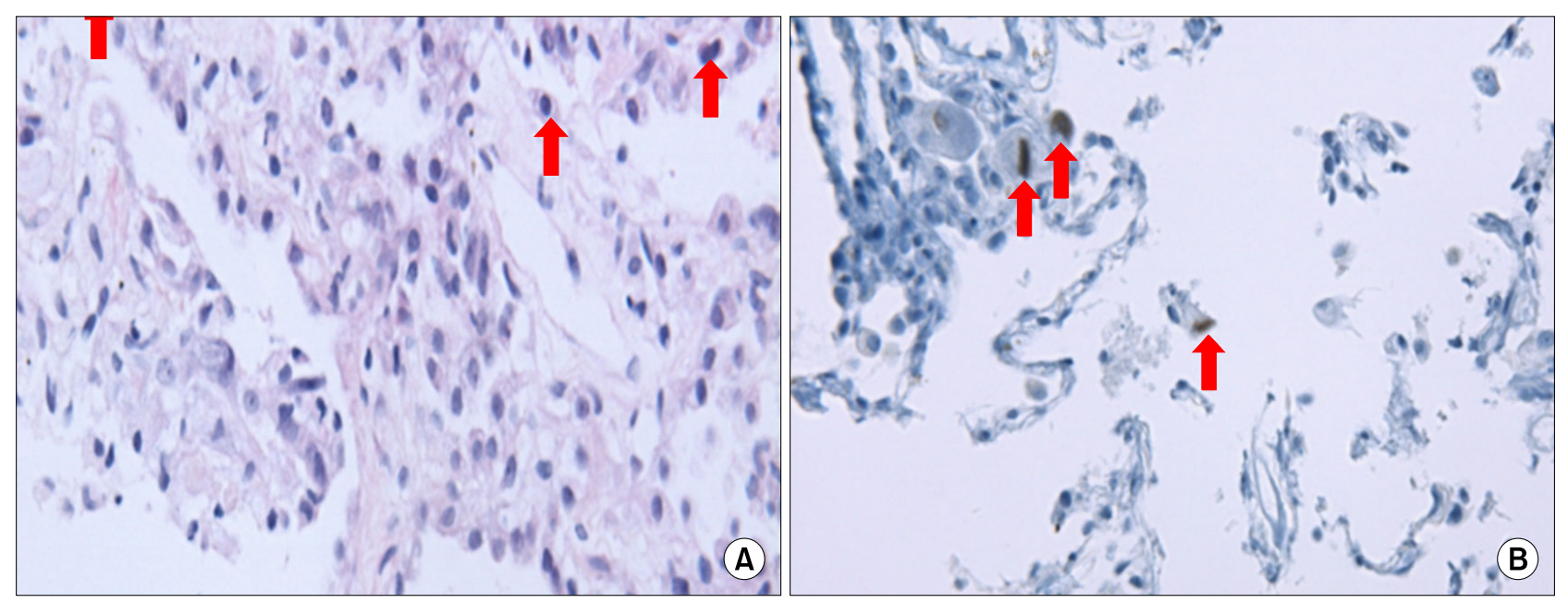

Figure 3. (A) The irregularly dilated alveoli showed mononuclear inflammatory cell infiltration in the interstitium and multiple intranuclear cytomegalovirus inclusions in the alveolar pneumonocytes (arrows) (H\&E, $\times 400)$. (B) Immunohistochemistry (IHC) with anti-cytomegalovirus (CMV) confirmed the CMV infected pneumonocytes with intranuclear inclusions (arrows) (IHC, $\times 400)$. 


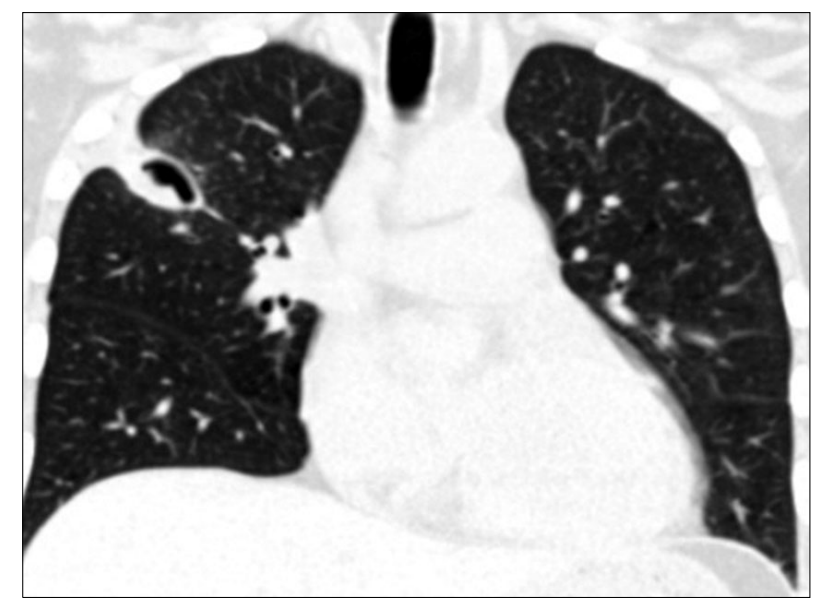

Figure 4. After treatment of cytomegalovirus pneumonia, the size of cavitary lung lesion was decreased.

nocytes (Figure 3A), and these inclusions showed positive reactions for $\mathrm{CMV}$ by immunohistochemistry (Figure 3B). Her treatment with reduction immunosuppressive drugs and administration of ganciclovir resulted in clinical improvement. Thereafter, cavitary lung lesion and pneumonia gradually subsided (Figure 4).

\section{DISCUSSION}

A major biological characteristic of CMV is its ability to become latent in the human host, with the potential for reactivation when a human is in an immunocompromised state, such as occurs with AIDS, immunosuppressive medication. Commonly, CMV is not thought to be the underlying cause of the cavitary lung lesions. Most of CMV pneumonias have ground-glass opacities, dense consolidation, and poorly defined nodular patterns on chest CT [3]. Cavitary lesions are more common in lung cancer, tuberculosis, pulmonary abscess, fungus and Wegener's granulomatosis than CMV pneumonia. One traditional method used to classify cavitary lesions is wall thickness. Cavities with a maximum wall thickness of $4 \mathrm{~mm}$ or less are usually nonmalignant and more than $15 \mathrm{~mm}$ are usually malignant [4]. In this case, the patient had weak evidences of inflammatory reactions in terms of CRP level and lupus disease activity despite fever. Other tests for infection were all negative. The radiologic finding was not correlated to usual manifestation of CMV pneumonia even though CMV infection was confirmed. Also, malignancy should be excluded. There are only few reports about cavitary CMV pneumonia. In USA, there were two case reports of cavitary pneumonia caused by acute CMV infection (positive CMV IgM) in SLE patients which presented with typical radiologic manifestation of CMV pneumonia and confirmed by wedge resection [5]. A case showed single cavitary lesion without fever and diagnosed by transbronchial lung biopsy (TBLB) [6]. In Japan, a SLE patient with single cavitary lesion was diagnosed by CMV antigenemia only [7]. Another SLE patient combined usual manifestation image was diagnosed by TBLB and CMV antigenemia and treated with additional $\gamma$ globulin [8]. In Korea, a SLE patient had usual image patterns and confirmed by open lung biopsy with positive CMV IgM [9]. Another SLE patient had also usual image patterns and confirmed by CMV PCR, TBLB and bronchoalveolar lavage [10]. When comparing with others, this SLE patient was confirmed by CT guided TNB and showed single cavitary lesion without other usual manifestations. She had improved by reduction of immunosuppressive drug and injection of ganciclovir without $\gamma$-globulin. Serologic confirmation is crucial to the diagnosis of CMV infection. Pathologic confirmation is much important to the diagnosis of CMV pneumonia. The radiographic appearance can sometimes be useful to differentiate among a broad spectrum of etiologies but should be combined with other data to obtain an accurate diagnosis. In that point, our patient got a final diagnosis properly by serologic and pathologic methods. By doing this, she could have a good prognosis with CMV pneumonia.

\section{SUMMARY}

We think this case is very rare case report in SLE patient with CMV pneumonia with cavitary lesions in Korea. We wish to share our experience which cavitary lung lesion as an unusual presentation with CMV pneumonia confirmed by CT guided lung biopsy and treated promptly and properly showing good prognosis. It should be recognized that CMV pneumonia can presented with cavitary lesions in the lungs of immunocompromised hosts such as patients who are taking immunosuppressive drugs.

\section{CONFLICT OF INTEREST}

No potential conflict of interest relevant to this article was reported. 


\section{REFERENCES}

1. Juárez M, Misischia R, Alarcón GS. Infections in systemic connective tissue diseases: systemic lupus erythematosus, scleroderma, and polymyositis/dermatomyositis. Rheum Dis Clin North Am 2003;29:163-84.

2. Leland DS, Emanuel D. Laboratory diagnosis of viral infections of the lung. Semin Respir Infect 1995;10:189-98.

3. Kang EY, Patz EF Jr, Müller NL. Cytomegalovirus pneumonia in transplant patients: CT findings. J Comput Assist Tomogr 1996;20:295-9.

4. Gadkowski LB, Stout JE. Cavitary pulmonary disease. Clin Microbiol Rev 2008;21:305-33.

5. Najjar M, Siddiqui AK, Rossoff L, Cohen RI. Cavitary lung masses in SLE patients: an unusual manifestation of CMV infection. Eur Respir J 2004;24:182-4.

6. Karakelides H, Aubry MC, Ryu JH. Cytomegalovirus pneumonia mimicking lung cancer in an immunocompetent host. Mayo Clin Proc 2003;78:488-90.

7. Katagiri A, Ando T, Kon T, Yamada M, Iida N, Takasaki Y. Cavitary lung lesion in a patient with systemic lupus erythematosus: an unusual manifestation of cytomegalovirus pneumonitis. Mod Rheumatol 2008;18:285-9.

8. Azuma N, Hashimoto N, Yasumitsu A, Fukuoka K, Yokoyama K, Sawada H, et al. CMV infection presenting as a cavitary lung lesion in a patient with systemic lupus erythematosus receiving immunosuppressive therapy. Intern Med 2009;48:2145-9.

9. Lee DH, Kim JW, Shin DH, Oh MD, Song YW, Choi KW, et al. A case of cytomegalovirus pneumonitis in a patient with systemic lupus erythematosus. Korean J Med 1999;56:103-7.

10. Han SH, Sohn YJ, Park MA, Lee S, Ryu SH, Lim TH, et al. A case of cytomegalovirus pneumonia and retinitis in a patients with systemic lupus erythematosus. J Korean Rheum Assoc 2003;10:456-61. 